http://jmscr.igmpublication.org/home/ ISSN (e)-2347-176x ISSN (p) 2455-0450 crossref DOI: https://dx.doi.org/10.18535/jmscr/v8i5.05

\author{
Journal Of Medical Science And Clinical Research \\ IGM Publication \\ An Official Publication of IGM Publication
}

\title{
Study of Enalapril and Diclofenac Sodium in Osteoarthritic Hypertensive Patients
}

\author{
Authors \\ S K Goswami, S S Goswami, C R Patil, D D Santani \\ Department of Pharmacology, L.M. College of Pharmacy, Navrangpura, Amedabad-380009, Gujarat \\ *Corresponding Author \\ Dr Sunita Goswami, M.Pharm PhD
}

Associate Professor, Department of Pharmacology, L.M. College of Pharmacy, Navarangpura, Amedabad-380009, Gujarat, INDIA

\begin{abstract}
Aim: The present study was designed to evaluate the pharmacodynamic drug-drug interaction between enalapril and concomitantly administered diclofenac sodium in osteoarthritic hypertensive [with or without type 2 diabetes mellitus (T2DM)] patients.

Materials and Methods: Total of 67 patients were randomized to either only enalapril $10 \mathrm{mg}$ or concomitant treatment of enalapril $10 \mathrm{mg}$ and diclofenac sodium $100 \mathrm{mg}$ for $8-12$ weeks in diseased states of hypertension and osteoarthritis with or without T2DM. Patients were assessed for systolic BP (SBP), diastolic $B P(D B P)$, insulin sensitivity, urinary albumin excretion rate (UAER), serum sodium $\left(S\right.$. $\left.\mathrm{Na}^{+}\right)$, serum potassium $\left(S . K^{+}\right)$, serum creatinine $\left(S_{c r}\right)$, creatinine clearance $(C L C R)$ and blood urea nitrogen $(B U N)$.

Results and Discussion: Baseline demographics and characteristics of the patients were comparable amongst all the groups. Adequately controlled antihypertensive effect of enalapril was significantly attenuated by diclofenac sodium when concomitantly administered with enalapril among diabetic (SBP: $p=0.004 ; D B P: p=0.007)$ and non-diabetic (SBP: $p=0.007 ; D B P: p=0.00006)$ pool of the patients. Insulin sensitivity was improved $(p=0.001)$ and UAER $(p=0.04)$ was better controlled among enalapril treated diabetic patients, while these effects were attenuated in diabetic patients receiving enalapril and diclofenac sodium concomitantly. Serum $\mathrm{Na}^{+}$and $\mathrm{S} . \mathrm{K}^{+}$levels were reduced and raised significantly in diabetic $\left(S . N a^{+}: p=0.02 ; S . K^{+}: p=0.002\right)$ as well as and non-diabetic $\left(S . N a^{+}: p=0.013 ; S . K^{+}: p=0.03\right)$ patients, respectively. In non-diabetic pool, $S_{c r}, C L C R$ and BUN were significantly increased $(p=0.002)$, lowered $(p<0.000001)$ and raised $(p=0.006)$, respectively, with enalapril and diclofenac sodium concomitant treatment. BUN was significantly increased $(p=0.014)$.

Conclusion: With the attenuation of antihypertensive efficacy, the chronic concomitant treatment of oral diclofenac sodium with enalapril leads to deterioration of insulin sensitivity (in T2DM patients), serum electrolytes and renal function in osteoarthritic hypertensive patients (with or without T2DM).

Keywords: diclofenac sodium; hypertension; enalapril, osteoarthritis, type-2 diabetes mellitus.
\end{abstract}

\section{Introduction}

Hypertension, a recognized risk factor in the development of cerebrovascular disorders, heart disease and renal failure, is a common disorder worldwide. Diseased states of hypertension and diabetes mellitus often exist together with 
hypertensive patients who are 2.5 times more prone to development of type 2 diabetes mellitus $(\mathrm{T} 2 \mathrm{DM})^{(1-2)}$. Angiotensin converting enzyme inhibitors (ACEIs) are drug of choice for T2DM patients with chronic kidney disease (CKD) as they delay the deterioration in glomerular filtration rate (GFR) and worsens albuminuria ${ }^{(3,4)}$.

Osteoarthritis is the most common degenerative joint disease. Besides paracetamol, non-steroidal anti-inflammatory drugs (NSAIDs) are widely used non-opioid analgesics for the treatment of osteoarthritis $^{(5-6)}$. Representing familiar cardiovascular co-morbid conditions, hypertension (with or without diabetes mellitus) and osteoarthritis are common conditions that increase in prevalence with age and often co-exist in same patients ${ }^{(7)}$ and lead to concomitant administration of antihypertensive medications and NSAIDs. Pharmacodynamically, NSAIDs attenuate the efficacy of antihypertensive medications with increase in BP mainly through salt and water retention ${ }^{(8,9)}$. Therefore, while choosing therapy for osteoarthritis in hypertensive patients, careful considerations include the effects of chosen analgesic on BP, interactions with antihypertensive agent(s) and effects on overall cardiovascular risk. Profound pharmacodynamic drug-drug interactions have been reported in previous studies involving NSAIDs like rofecoxib, aspirin, indomethacin, ibuprofen, diclofenac, celecoxib or piroxicam and ACEIs ${ }^{(10-}$ 15)

Enalapril, an orally administered prodrug hydrolyzed to form the active metabolite enalaprilat $^{(16)}$, is widely used ACEI in hypertensive patients. Diclofenac sodium, is a potent inhibitor of PGs and thromboxane (THX) synthesis via non-selective inhibition of COX-1 and $\mathrm{COX}-2^{(17)}$. It is one of the most frequently used NSAIDs among osteoarthritic patients.

In the light of above reports, the present study was designed to assess the pharmacodynamic drugdrug interaction between enalapril and diclofenac sodium when taken concomitantly in osteoarthritic hypertensive (with or without T2DM) patients based on evaluation of $\mathrm{BP}$, insulin sensitivity, renal function and serum electrolytes levels.

\section{Material and Methods Study Design}

The present study was an open label, multipledose, randomized, parallel group, single-centre trial conducted at out-patient department (OPD) of Pramukhswami Healthcare \& Research Centre, Ahmedabad, INDIA in patients with mild to moderate essential hypertension (with or without T2DM) and osteoarthritis. The study protocol and informed consent form were approved by an independent ethics committee formed in compliance with the ICH-GCP guidelines. Written informed consent was obtained from each of the patient or its legally acceptable representative before their participation in the study.

\section{Study Patients}

67 patients of both genders aged 40-70 years with mild to moderate essential hypertension [diastolic blood pressure (DBP) of 95 to $114 \mathrm{~mm} \mathrm{Hg}$; with or without T2DM] and osteoarthritis were enrolled on outpatient basis. Patients included were without the histories of coronary insufficiency, myocardial infarction, congestive heart failure, moderate renal impairment or failure (i.e. serum creatinine not more than $1.5 \mathrm{mg} / \mathrm{dl}$ ) and cerebrovascular accident or without gross abnormalities in routine hematological and biochemical parameters at the time of enrollment. At the time of screening, patients were excluded if they had secondary or severe hypertension, joint disorders other than osteoarthritis or history of chronic skin rash. Women patients required to be were non-pregnant, non-lactating, or not susceptible to pregnancy (i.e. surgically sterile, or on a reliable contraceptive measure or attained menopause for one year). Patients were also excluded if they were on an ACEI in the past or at the time of enrolment or had the hypersensitivity to ACEIs or NSAIDs.

The patients with T2DM were on oral hypoglycaemics for $\geq 2$ years before enrolment into the study. Osteoarthritic patients received 
symptomatic treatment on "whenever required" basis. Diagnosis of essential hypertension was based on a family history of high BP and the absence of reversible causes as assessed by appropriate screening test(s).

\section{Study Methodology}

Before initiation of the study, the health status of each patient was evaluated on the basis of history and physical examination, with special emphasis on examination of the cardiovascular system and other organs commonly damaged by hypertension. Initial laboratory tests included complete blood count, serum sodium $\left(\mathrm{S} . \mathrm{Na}^{+}\right)$, serum potassium $\left(\mathrm{S} . \mathrm{K}^{+}\right.$), blood urea nitrogen $(\mathrm{BUN})$, serum creatinine $\left(\mathrm{S}_{\mathrm{cr}}\right)$, creatinine clearance (CLCR), fasting blood sugar and lipid profile. The clinical history of each patient including BP recordings, previous disease histories, drug regimen and other positive symptoms were documented in case report forms (designed as per the regulatory requirements). Each patients were randomized to one of the treatment groups as per the table-1.

Supine BP and heart rates were measured twice at each visit, and the mean of two BP recordings was recorded. Supine measurements were made after the patient was recumbent for 5 minutes. All measurements were made on the same arm, usually the right. All the parameters were recorded before and after the treatment (8-12 weeks).

\section{Sample Analysis}

The blood samples were analysed for blood sugar [Glucose Oxidase - Peroxidase (GOD-POD) Method, End Point; SPAN Diagnostics Ltd., Surat, India], BUN [Diacetyl monoxime (DAM) method, SPAN Diagnostics Ltd., Surat, India], $\mathrm{S}_{\mathrm{cr}}$ [Alkaline picrate method; SPAN Diagnostics Ltd., Surat, India], $\mathrm{S} . \mathrm{Na}^{+}$[Flame photometry] and $\mathrm{S}$. $\mathrm{K}^{+}$[Flame photometry]. Insulin sensitivity index ( $\mathrm{K}_{\text {ITT }}$ ) was calculated as per the method of Alford et $a l^{(24)}$ in which estimated blood glucose was plotted against time on semi log paper to calculate $t_{1 / 2}$ and insulin sensitivity index was calculated by equation-1 given below. CLCR was calculated using Cockcroft-Gault equation (18), as given below in equation-2.

$\mathrm{K}_{\mathrm{ITT}}=\left(0.693 / \quad \mathrm{t}_{1 / 2}\right)$
$\ldots \ldots \ldots \ldots \ldots \ldots \ldots \ldots \ldots \ldots \ldots \ldots \ldots$

$\mathrm{CLCR}_{\text {male }}=[(140$-age $) *$ body weight $] /\left(72 * \mathrm{~S}_{\mathrm{cr}}\right)$ (Equation-2)

[Note: The proportion of muscle mass on body weight is relatively lower in women than in men. Therefore, the calculated value of CLCR was multiplied by the factor of 0.85 in female patients; age: years; body weight: $\mathrm{kg} ; \mathrm{S}_{\mathrm{cr}}$ : $\mathrm{mg} / \mathrm{dL}$; CLCR: $\mathrm{ml} / \mathrm{min}$ ]

Overnight urine (early-morning) was collected before and after the treatment period. Urine samples were estimated for urinary albumin excretion rate (UAER; Micral test; paper chromatography; Boehringer Mannheim India Ltd., Mumbai, India).

\section{Statistical Analysis}

Data were expressed as the mean \pm SEM. For all the parameters, one-way analysis of variance (ANOVA) was used to assess the differences among the treatment groups. Otherwise, the equivalent non-parametric, Wilcoxon two-sample rank sum test was applied. A 'p' value of less than $5 \% \quad(\mathrm{p}<0.05)$ was considered to indicate significant differences between the means. Further, 95\% confidence interval was calculated for differences of means (Treatment-Baseline) for each parameter.

\section{Results}

Before enrolment, patients were evaluated for baseline demographics and characteristics like age, sex, height, weight, biochemical parameters, haematological parameters, risk factors involved and underlying disease. The mean age of patients was 56.5 years. Male and female patients represented $44.8 \%$ and $55.2 \%$ proportionate among total pool of the patients. Gender distribution along with the distribution of diabetic and non-diabetic patients in each group is depicted 
in table-1. Among total number of patients, 44 (65.7\%), 19 (28.4\%) and $04(5.9 \%)$ patients were following sedentary, moderate and heavy lifestyles, respectively. A total of 21 patients $(51.2 \%)$ were suffering from obesity where as 19 (43.2\%) patients had family histories of mild or moderate hypertension. Baseline demographics and characteristics of the patients included were comparable amongst the groups.

Physical parameters like SBP, DBP and biochemical parameters viz. insulin sensitivity and UAER (in diabetic patients only), $\mathrm{S}_{\mathrm{cr}}$, CLCR, BUN, S. $\mathrm{Na}^{+}$and S. $\mathrm{K}^{+}$were studied in nondiabetic and diabetic patients. Results have been represented by mean differences of changes as shown in the tables 2-3.

\section{Blood Pressure}

The BP was adequately controlled (i.e. DBP $\leq 90$ $\mathrm{mmHg}$ ) in enalapril administered diabetic as well as non-diabetic pool of the patients as compared to their baseline values. However, the BP lowering effect of enalapril was significantly attenuated by concomitantly administered diclofenac sodium in diabetic (SBP: $\mathrm{p}=0.004$; DBP: $\mathrm{p}=0.007)$ and non-diabetic $(\mathrm{p}=0.007$; DBP: $\mathrm{p}=0.00006$ ) patients.

\section{Insulin Sensitivity and Urinary Albumin Excretion Rate}

Insulin sensitivity and UAER were studied in diabetic patients only. Insulin sensitivity was increased significantly in enalapril $(p=0.001)$ treated patients in comparison to patients receiving enalapril and diclofenac sodium concomitantly. UAER (>20 mg/day at baseline) decreased significantly in enalapril $(\mathrm{p}=0.04)$ treated patients when compared with patients receiving enalapril and diclofenac sodium concomitantly.

\section{Serum Electrolytes}

$\mathrm{S}$. $\mathrm{Na}^{+}$and $\mathrm{S} . \mathrm{K}^{+}$levels were respectively, reduced and raised significantly in diabetic ( $\mathrm{S} . \mathrm{Na}^{+}$: $\mathrm{p}=0.02 ; \mathrm{S} . \mathrm{K}^{+}: \mathrm{p}=0.002$ ) as well as and nondiabetic $\left(\mathrm{S} . \mathrm{Na}^{+}: \mathrm{p}=0.013 ; \mathrm{S} . \mathrm{K}^{+}: \mathrm{p}=0.03\right)$ patients receiving concomitant treatment of enalapril and diclofenac sodium in comparison with enalapril administered patients. Although S. $\mathrm{Na}^{+}$(Normal physiological range: $135-144 \mathrm{mEq} / \mathrm{L}$ ) and $\mathrm{S} . \mathrm{K}^{+}$ (Normal physiological range: $3.6-4.8 \mathrm{mEq} / \mathrm{L}$ ) levels were lowered and raised, respectively, in patients receiving either enalapril or concomitant treatments of enalapril and diclofenac sodium, none of the patients developed any clinical sign of hyponatremia or hyperkalemia, respectively.

\section{Effect on Renal Function}

Renal function of the patients was studied in terms of $S_{c r}$, CLCR and BUN. $S_{\text {cr }}$ levels were found within normal range at baseline (or post-washout) and remained within that range in patients receiving enalapril therapy alone. Among nondiabetic pool, $\mathrm{S}_{\mathrm{cr}}$ levels were significantly increased $(p=0.002)$ in patients receiving concomitant treatment of enalapril and diclofenac sodium when compared to enalapril treated patients. However this trend was not followed in case of diabetic patients $(\mathrm{p}=0.08)$ when the two treatments were compared. As evident from the results, $S_{\mathrm{cr}}$ levels were relatively higher in nondiabetic patients compared with diabetic patients receiving concomitant treatment of enalapril and diclofenac sodium.

Among non-diabetic pool, CLCR was reduced significantly $\quad(p<0.000001) \quad$ in patients concomitant treatment of enalapril and diclofenac sodium when compared to enalapril treated patients. However, it was significantly increased $(\mathrm{p}<0.000001)$ in enalapril and diclofenac sodium treatment concomitantly as compared to enalapril receiving patients among diabetic pool.

BUN levels were significantly increased (diabetic pool: $\mathrm{p}=0.014$; non-diabetic pool: $\mathrm{p}=0.006$ ) and reached towards upper limit of physiological range in patients receiving concomitant treatment of enalapril and diclofenac sodium when compared to enalapril treated patients. 
Table-1: Study Medications and their duration

\begin{tabular}{|c|c|c|c|c|c|}
\hline Group & Diseased state & $\mathbf{N}$ & Sex & $\begin{array}{l}\text { Treatments } \\
\text { Dose/day }\end{array}$ & $\begin{array}{c}\text { Duration of } \\
\text { therapy }\end{array}$ \\
\hline 1 & $\mathrm{HT}+\mathrm{OA}$ & 17 & $\begin{array}{l}\text { M: } 08 \\
\text { F: } 09\end{array}$ & Enalapril10mg & $8-12$ weeks \\
\hline 2 & $\mathrm{HT}+\mathrm{OA}$ & 18 & $\begin{array}{l}\text { M: } 07 \\
\text { F: } 11\end{array}$ & $\begin{array}{c}\text { Enalapril 10mg }+ \\
\text { Diclofenac sodium } 100 \mathrm{mg}\end{array}$ & $8-12$ weeks \\
\hline 3 & $\mathrm{HT}+\mathrm{OA}+\mathrm{T} 2 \mathrm{DM}$ & 16 & $\begin{array}{l}\text { M: } 08 \\
\text { F: } 08\end{array}$ & Enalapril 10mg & $8-12$ weeks \\
\hline 4 & $\mathrm{HT}+\mathrm{OA}+\mathrm{T} 2 \mathrm{DM}$ & 16 & $\begin{array}{l}\text { M: } 07 \\
\text { F: } 09\end{array}$ & $\begin{array}{c}\text { Enalapril } 10 \mathrm{mg}+ \\
\text { Diclofenac sodium } 100 \mathrm{mg}\end{array}$ & $8-12$ weeks \\
\hline
\end{tabular}

N: number of patients; HT: Hypertension; OA: Osteoarthritis; M: male; F: female; T2DM: Type 2 diabetes mellitus

Table-2: The mean differences of changes $( \pm$ SEM) with $95 \%$ confidence interval $(\mathrm{CI})$ in the parameters studied in diabetic patients treated with enalapril $(\mathrm{E})$ and enalapril-diclofenac sodium combination $(\mathrm{E}+\mathrm{D})$

\begin{tabular}{|l|c|c|c|c|}
\hline Parameters & $\mathbf{E}(\mathbf{n}=\mathbf{1 6})$ & $\mathbf{E}+\mathbf{D}(\mathbf{n}=\mathbf{1 6})$ & $\boldsymbol{p}$-values & $\mathbf{C I} \mathbf{( 9 5 \% )}$ \\
\hline $\mathrm{SBP}(\mathrm{mm} \mathrm{Hg})$ & $-18.25 \pm 3.91$ & $0.01 \pm 4.44$ & 0.004 & $-30.19,-6.31$ \\
\hline DBP $(\mathrm{mm} \mathrm{Hg})$ & $-19.00 \pm 10.55$ & $-9.31 \pm 8.31$ & 0.007 & $-16.47,-2.91$ \\
\hline Insulin sensitivity & $0.24 \pm 0.03$ & $0.05 \pm 0.04$ & 0.001 & $0.08,0.30$ \\
\hline UAER $(\mathrm{mg} / \mathrm{day})$ & $-17.56 \pm 2.54$ & $-8.31 \pm 3.44$ & 0.04 & $-17.89,-0.61$ \\
\hline $\mathrm{S} . \mathrm{Na}^{+}(\mathrm{mEq} / \mathrm{L})$ & $-3.38 \pm 1.72$ & $-9.69 \pm 1.87$ & 0.02 & $1.17,11.45$ \\
\hline $\mathrm{S} . \mathrm{K}^{+}(\mathrm{mEq} / \mathrm{L})$ & $0.13 \pm 0.16$ & $0.82 \pm 0.12$ & 0.002 & $-1.10,-0.28$ \\
\hline $\mathrm{S}_{\mathrm{cr}}(\mathrm{mg} / \mathrm{dL})$ & $0.11 \pm 0.08$ & $-0.11 \pm 0.08$ & 0.08 & $-0.02,0.46$ \\
\hline $\mathrm{CLCR}(\mathrm{ml} / \mathrm{min})$ & $-7.76 \pm 2.73$ & $8.77 \pm 3.78$ & $<0.000001$ & $-11.27,12.29$ \\
\hline BUN $(\mathrm{mg} / \mathrm{dL})$ & $3.19 \pm 1.23$ & $7.44 \pm 1.08$ & 0.014 & $-7.56,-0.94$ \\
\hline
\end{tabular}

SBP: Systolic Blood Pressure; DBP: Diastolic Blood Pressure; UAER: Urinary Albumin Excretion Rate; S. Na ${ }^{+}$: sodium; $\mathrm{S}^{+} \mathrm{K}^{+}$: Serum potassium; $\mathrm{S}_{\mathrm{cr}}$ : Serum creatinine; CLCR: Creatinine clearance; BUN: Blood Urea Nitrogen;

Table-3: The mean differences of changes $( \pm$ SEM) with $95 \%$ confidence interval $(\mathrm{CI})$ in the parameters studied in non-diabetic patients treated with enalapril $(\mathrm{E})$ and enalapril-diclofenac sodium combination $(\mathrm{E}+\mathrm{D})$

\begin{tabular}{|l|c|c|c|c|}
\hline Parameters & $\mathbf{E}(\mathbf{n}=\mathbf{1 7})$ & $\mathbf{E}+\mathbf{D}(\mathbf{n}=\mathbf{1 8})$ & $\boldsymbol{p}$-values & $\mathbf{C I}(\mathbf{9 5 \%})$ \\
\hline $\mathrm{SBP}(\mathrm{mm} \mathrm{Hg})$ & $-17.88 \pm 3.52$ & $-2.11 \pm 4.14$ & 0.007 & $-26.91,-4.63$ \\
\hline $\mathrm{DBP}(\mathrm{mm} \mathrm{Hg})$ & $-16.65 \pm 2.23$ & $-4.72 \pm 2.21$ & 0.00006 & $-18.34,-5.52$ \\
\hline $\mathrm{S} . \mathrm{Na}^{+}(\mathrm{mEq} / \mathrm{L})$ & $-2.0 \pm 1.70$ & $-8.17 \pm 1.64$ & 0.013 & $1.35,10.99$ \\
\hline $\mathrm{S} . \mathrm{K}^{+}(\mathrm{mEq} / \mathrm{L})$ & $0.16 \pm 0.15$ & $0.63 \pm 0.14$ & 0.03 & $-0.88,-0.06$ \\
\hline $\mathrm{S}_{\mathrm{cr}}(\mathrm{mg} / \mathrm{dL})$ & $0.08 \pm 0.07$ & $0.43 \pm 0.08$ & 0.002 & $-0.56,-0.14$ \\
\hline $\mathrm{CLCR}(\mathrm{ml} / \mathrm{min})$ & $-10.2 \pm 4.83$ & $-35.7 \pm 11.9$ & $<0.000001$ & $-39.3,-6.75$ \\
\hline BUN $(\mathrm{mg} / \mathrm{dL})$ & $3.82 \pm 0.82$ & $7.94 \pm 1.15$ & 0.006 & $-6.95,-1.29$ \\
\hline
\end{tabular}

SBP: Systolic Blood Pressure; DBP: Diastolic Blood Pressure; S. Na+ ${ }^{+}$Serum sodium; S. K ${ }^{+}$: Serum potassium; $\mathrm{S}_{\mathrm{cr}}$ : Serum creatinine; CLCR: Creatinine clearance; BUN: Blood Urea Nitrogen;

\section{Discussion}

In view of the prominent effects of NSAIDs on $\mathrm{BP}$ elevation or attenuating the BP reduction when administered concomitantly with ACEIs, we evaluated potential pharmacodynamic drug-drug interaction between concomitantly administered enalapril and diclofenac sodium in hypertensive (with or without T2DM) osteoarthritic patients.
Antihypertensive activity of ACEIs is not only confined to the inhibition of Angiotensin-II (AngII), but also to the ACEI mediated increased local formation of bradykinin. Bradykinin acts on bradykinin receptors in the vascular endothelium to produce potential vasodilating mediators, PGs and nitric oxide ${ }^{(19)}$. PGs act locally in the kidney to maintain homeostasis by regulating sodium and 
water reabsorption, particularly in the thick ascending loop of Henle and the collecting duct. NSAIDs inhibit the COX enzyme leading to systemically reduction in the production of thromboxane $\mathrm{A}_{2}$ and vasodilatory PGs. At the renal level, the inhibition of PGs results in a drop in the renal blood flow, with reduced GFR and consequent rise in urea and creatinine. Inhibition of PGs may also trigger an increase in chloride absorption, with consequent sodium retention, edema and hypertension. The reduction of PGs may induce a reduction of renin and aldosterone with consequent potassium retention and hyperkalemia. Finally, the reduction in PGs leads to an increase in the effect of antidiuretic hormone $(\mathrm{ADH})$, which contributes to water retention with hyponatremia $^{(20)}$. Thus NSAID mediated salt retention and reduced GFR may lead to elevation in $\mathrm{BP}$ or may worsen the pre-existing hypertension. As shown in previous clinical studies, NSAIDs like rofecoxib, aspirin, indomethacin, ibuprofen, diclofenac, celecoxib or piroxicam have remarkably blunted the BP control efficacy of ACEIs ${ }^{(11-15)}$ in hypertensive and/or osteoarthritic patients. Also, meta-analysis of number of clinical studies revealed that indomethacin, naproxen, piroxicam and ibuprofen cause greater rise in BP among hypertensive patients.

In the present study, enalapril reduced SBP and DBP remarkably in diabetic as well as nondiabetic patients. However, BP control was lost significantly when enalapril was concomitantly administered with diclofenac sodium. The attenuation of the antihypertensive efficacy of enalapril by diclofenac sodium observed in our study is also in agreement with available reports as discussed earlier. Therefore, due monitoring of $\mathrm{BP}$ is recommended during concomitant administration of ACEI and NSAID.

Insulin resistance accompanied by metabolic changes like hyperinsulinaemia, glucose intolerance and dyslipidaemia represent independent risk factors for coronary heart disease and are more common in hypertensive patients than the normotensive ones ${ }^{(19)}$. In patients with T2DM, insulin resistance leads to poor glycaemic control (impaired fasting glucose), hyperinsulinaemia, hypertension, dyslipidemia and possibly other pathological conditions which are constellating factors of metabolic syndrome ${ }^{(20)}$.

In the present study, insulin sensitivity was measured as glucose disposal rate, $\mathrm{K}_{\mathrm{ITT}}$, by the method of Alford et $a l^{(21)}$. Insulin sensitivity enhanced significantly in patients receiving chronic treatment with enalapril which was in accordance with the previous studies of enalapril which showed remarkable increase in insulin sensitivity and improvement in endothelial function among essential hypertensive patients. The proposed mechanism behind the beneficial effects of ACEIs on improvement of insulin sensitivity and insulin secretion is through the vascular system and ionic balance. ACEIs cause vasodilation which induces improved blood circulation in skeletal muscle and pancreas leading to peripheral insulin action and promoting insulin secretion, respectively. By preserving potassium and magnesium pools with the blockade of aldosterone, ACEIs could improve cellular insulin secretion and action. Another important mechanism which has been described is the ACEI mediated inhibition of angiotensin and bradykinin enhancement which play an important role in insulin cascade signaling and increase in glucose transporter type-4 (GLUT-4) ${ }^{(22)}$.

Further, concomitant treatment of diclofenac sodium with enalapril counteracted the enalapril mediated enhancement of insulin sensitivity. Chronic inflammation is an important risk factor involved in the pathophysiology of insulin resistance. NSAIDs like salsalate, sodium salicylate and indomethacin have been reported to improve insulin resistance in clinical as well as pre-clinical models of T2DM (23-25). However, diclofenac sodium did not show any improvement in insulin resistance during the present study. Therefore, findings of our study are suggestive of the fact that hypertensive patients with diabetes 
and osteoarthritis receiving long-term treatment with enalapril, oral hypoglycaemics and NSAIDs must be closely monitored for dose adjustments in maintaining optimum glycaemic control.

Chronic kidney disease (CKD) associated with diabetes is defined by the presence of microalbuminuria (20 to $200 \mu \mathrm{g} / \mathrm{min}$ ) or macroalbuminuria $(>300 \mu \mathrm{g} / \mathrm{min})$ or proteinuria and occurs within 20 to $25 \mathrm{yr}$ of the onset of type 1 or type 2 diabetes in a large proportion of patients. Hypertension and diabetes are coexisting states involved in the pathophysiology of CKD. Approximately $30 \%$ of patients with diabetic nephropathy, a chronic kidney disease, progress to end stage renal disease (ESRD) ${ }^{(26)}$. The control of each $10 \mathrm{~mm} \mathrm{Hg}$ of SBP significantly reduces overall mortality associated with diabetic nephropathy and the progression of diabetic nephropathy to ESRD with significant reductions in the proteinuria.

In the present study, UAER was reduced following chronic treatment with enalapril in diabetic hypertensive patients. As reported in the previous studies, NSAIDs have shown antiproteinuric effects which are attributed to reduction in the effective renal plasma flow (ERPF) and GFR by inhibiting $\mathrm{PGE}_{2}$ production $^{(27)}$. However, UAER reduction effect of enalapril was attenuated by concomitantly administered diclofenac sodium in diabetic hypertensive and arthritic patients. Thus, the findings of our study advocate appropriate monitoring of UAER in those patients who are receiving chronic treatment with enalapril and diclofenac sodium concomitantly.

Hyperglycemia and hyperinsulinaemia directly affect renal tubule leading to inhibition of sodium excretion . Also renin-angiotensin-aldosterone pathway leads to sodium retention. By contrast, natriuresis occurs soon after initiation of ACEI therapy ${ }^{(28)}$. In present study, there was no significant change in $\mathrm{S}$. $\mathrm{Na}^{+}$levels following enalapril treatment in diabetic as well as nondiabetic patients even though there was a decrease in $\mathrm{S}$. $\mathrm{Na}^{+}$levels. Fluid retention is the most common NSAID-related renal complication, occurring to some degree in virtually all exposed individuals and is readily reversible on discontinuation of the NSAID ${ }^{(29)}$. In our study, S. $\mathrm{Na}^{+}$levels were significantly reduced in the patients receiving concomitant treatment of enalapril and diclofenac sodium which is beneficial effect as far as anti-hypertensive effect is concerned. Although these observations are not clinically significant as values of $\mathrm{S}$. $\mathrm{Na}^{+}$level were falling within the normal range, one cannot rule out hyponatraemic effect in the group of patients receiving long-term therapy with diclofenac sodium and enalapril.

In addition, S. $\mathrm{K}^{+}$levels increased in patients receiving chronic enalapril therapy when compared with the baseline values and in patients receiving chronic concurrent treatment of enalapril and diclofenac sodium among diabetic and non-diabetic pools. However, none of the patients developed any sign of hyperkalemia. ACEIs are known to reduce aldosterone secretion and potassium retention is not uncommon during treatment with such agents. NSAIDs are also reported to cause hyperkalemia ${ }^{(30)}$. Our observations are parallel with the earlier findings of hyperkalemia in patients receiving ACEIs and NSAIDs concomitantly. Thus, it could be suggested that patients must be monitored for $\mathrm{S}$. $\mathrm{K}^{+}$levels to avoid hyperkalemic complications such as fatigue, weakness, tingling, numbness, paralysis, bradycardia, palpitations, or difficulty in breathing.

The most common cause of an acute increase in $S_{\mathrm{cr}}$ levels with reduction in CLCR following inhibition of the renin angiotensin system, results from a decreased arterial blood volume. This is due to hypoperfusion secondary to volume depletion from overaggressive diuresis, low cardiac output seen in heart failure, or both. In these clinical settings, the reduced pressure head from the afferent arteriole further lessens the already reduced intraglomerular pressure imposed by ACEIs. Thus, the compensatory elevation of single-nephron GFR seen in renal insufficiency 
and diabetes condition is reduced in an almost additive manner when hypoperfusion and ACE inhibition coexist. This reduction in GFR is reversible within one month when ACEIs are stopped except in renal insufficient patients ${ }^{(31)}$. By inhibiting COX, NSAIDs systematically reduce the production of several PGs with vasodilating effect, including $\mathrm{PGE}_{2}$ and $\mathrm{PGI}_{2}$. At the renal level, inhibition of PGs results in a fall of renal blood flow, with reduced GFR and a consequent increase in serum urea, and $S_{\mathrm{cr}}$ with a decrease in $\mathrm{CLCR}^{(19)}$.

Non-diabetic patients receiving diclofenac sodium concomitantly with enalapril showed significant rise in $\mathrm{S}_{\mathrm{cr}}$ and BUN levels with significant fall in CLCR levels as compared to patients receiving enalapril treatment alone. This shows that GFR and blood flow to the kidneys reduced significantly in the patients receiving the concomitant treatment of diclofenac sodium and enalapril leading to deterioration of renal function. These findings are in agreement with the report of Bouvy et al. revealing an increased risk of hospitalization for renal dysfunction at the start of NSAID therapy in users of ACEIs ${ }^{(32)}$. Among diabetic pool of the patients, the concomitant treatment with enalapril and diclofenac sodium decreased $S_{\mathrm{cr}}$ and significantly increased CLCR which is in contrast to the earlier studies and requires further investigations.

Similar study has been conducted earlier in osteoarthritic hypertensive (with or without T2DM) patients and fetched almost similar detrimental influence of diclofenac sodium when administered concomitantly with lisinopril ${ }^{(33)}$. In addition to above findings, key issues include the use of oral NSAIDs (or diclofenac) in geriatric patients concomitantly receiving ACEIs (enalapril and lisinopril) and whether the use of topical NSAIDs is appropriate or not in hypertensive osteoarthritic patients. Geriatric patients have increased prevalence of cardiovascular complications with diminished renal function. Hence, prescribing NSAID alone or in combination with ACEI strictly requires thorough medical history check-up and monitoring at regular intervals. Patients with CLCR $<50 \mathrm{ml} / \mathrm{min}$ should not be prescribed NSAIDs as they have renal PG processes blunted by selective and nonselective NSAIDs. Topical NSAIDs are often used with the therapeutic rationale of minimizing systemic NSAID exposure, with a focus on those who have risk factors but require the therapeutic benefit of an anti-inflammatory agent. Further, it has also been shown in one of the study to have 150 -fold lower peak plasma concentration and 17fold lower mean plasma concentration using $1 \%$ diclofenac gel relative to a comparable dose of oral diclofenac ${ }^{(34)}$.

\section{Conclusion}

In conclusion, it can be suggested from our study that chronic co-administration of oral diclofenac sodium reduces the antihypertensive efficacy of the enalapril and may have deleterious effect on insulin sensitivity, serum electrolytes and renal function in hypertensive osteoarthritic patients (with or without T2DM). Thus, the prescribing physician should decide whether to add oral diclofenac sodium to the regimen of enalapril considering the risks and benefits and even if oral diclofenac sodium is added, appropriate monitoring of the parameters may prevent the deleterious effect of the combination.

\section{References}

1. Gress TW, Nieto FJ, Shahar E, Wofford MR, Brancati FL. Hypertension and antihypertensive therapy as risk factors for type 2 diabetes mellitus. Atherosclerosis Risk in Communities Study. N Engl J Med. 2000; 342(13):905-12.

2. Sowers JR, Bakris GL. Antihypertensive therapy and the risk of type 2 diabetes mellitus. N Engl J Med. 2000; 342: 96970.

3. Sowers JR, Haffner S. Treatment of cardiovascular and renal risk factors in the diabetic hypertensive. Hypertension 2002; 40:781-8. 
4. Gaede P, Vedel P, Larsen N, Jensen GVH, Parving $\mathrm{HH}$, Pedersen O. Multifactorial Intervention and Cardiovascular Disease in Patients with Type 2 Diabetes. N Engl J Med. 2003; 348(5):383-93.

5. Hunter DJ, Lo GH. The management of osteoarthritis: an overview and call to appropriate conservative treatment. Med Clin North Am. 2009; 93:127-43.

6. Mackenzie IS, MacDonald TM. Treatment of osteoarthritis in hypertensive patients. Expert Opin. Pharmacother. 2010; 11(3):393-403..

7. Singh G, Miller JD, Lee FH, Pettitt D, Russell MW. Prevalence of cardiovascular disease risk factors among US adults with self-reported osteoarthritis: data from the Third National Health and Nutrition Examination Survey. Am J Manag Care. 2002; 8(15-Suppl):S383-91.

8. Harris RC. Cyclooxygenase-2 Inhibition and Renal Physiology. Am J Cardiol 2002; 89(Suppl):10D-17D.

9. Whelton A, White WB, Bello AE, Puma JA, Fort JG, and the SUCCESS-VII Investigators. Effects of celecoxib and rofecoxib on blood pressure and edema in patients > or $=65$ years of age with systemic hypertension and osteoarthritis. Am J Cardiol 2002; 90(9):959-63.

10. Meune C, Mahe I, Mourad JJ, Simoneau G, Knellwolf AL, Bergmann JF, Caulin C. Interaction between angiotensinconverting enzyme inhibitors and aspirin: a review. Eur J Clin Pharmacol 2000; 56: 609-20.

11. Morgan TO, Anderson A, Bertram D. Effect of indomethacin on blood pressure in elderly people with essential hypertension well controlled on amlodipine or enalapril. Am J Hypertens. 2000; 13(11):1161-7.

12. Conlin PR, Moore TJ, Swartz SL, Barr E, Gazdick L, Fletcher C, DeLucca P, Demopoulos L. Effect of Indomethacin on
Blood Pressure Lowering by Captopril and Losartan in Hypertensive Patients. Hypertension 2000; 36:461-465.

13. Palmer R, Weiss R, Zusman RM, Haig A, Flavin S, MacDonald B. Effects of Nabumetone, Celecoxib, and Ibuprofen on Blood Pressure Control in Hypertensive Patients on Angiotensin Converting Enzyme Inhibitors. Am J Hypertens 2003; 16:135-9.

14. Izhar M, Alausa T, Folker A, Hung E, Bakris GL. Effects of COX Inhibition on blood pressure and kidney function in ACE Inhibitor-treated blacks and Hispanics. Hypertension 2004; 43:573-7.

15. Pavlicevic I, Kuzmanic M, Rumboldt M, Rumboldt Z. Interaction between antihypertensives and NSAIDs in primary care: a controlled trial. Can J Clin Pharmacol 2008; 15(3):e372-82.

16. Hair PI, Scott LJ, Perry CM. Fixed-dose combination Lercanidipine/Enalapril. Drugs 2007; 67(1):95-106.

17. McCormack PL, Scott LJ. Diclofenac Sodium Injection $\quad\left(\right.$ Dyloject $\left.^{\circledR}\right)$ : In Postoperative Pain. Drugs 2008; 68(1):123-30.

18. Stevens LA, Nolin TD, Richardson MM, Feldman HI, Lewis JB, Rodby R, Townsend R, Okparavero A, Zhang YL, Schmid CH, Levey AS, MD [on behalf of the Chronic Kidney Disease Epidemiology Collaboration (CKD-EPI)]. Comparison of Drug Dosing Recommendations Based on Measured GFR and Kidney Function Estimating Equations. Am J Kidney Dis. 2009; 54(1):33-42.

19. Bray GA, Bellanger T. Epidemiology, Trends and Morbidities of Obesity and the Metabolic Syndrome. Endocrine 2006; 29(1):109-17.

20. Yavuz D, Koc M , Toprak A, Akpinar I, Velioglu A, Deyneli O, Haklar G, Akalin S. Effects of ACE inhibition and AT1receptor antagonism on endothelial 
function and insulin sensitivity in essential hypertensive patients. Journal of the Renin-Angiotensin-Aldosterone System 2003; 4:197-203.

21. Alford FP, Martin FIR, Pearson MJ. The significance and interpretation of mildly abnormal oral glucose tolerance. Diabetologia 1971; 7:173-80.

22. Rumore MM, Kim KS. Potential role of salicylates in type 2 diabetes. Ann. Pharmacother. 2010; 44(7-8):1207-21.

23. Fleischman A, Shoelson SE, Bernier R, Goldfine AB. Salsalate Improves Glycemia and Inflammatory Parameters in Obese Young Adults. Diabetes Care 2008; 31(2):289-94.

24. Bing He, Sheng Zhao, Wei Zhang, Yan Li, Ping Han. Effect of sodium salicylate on oxidative stress and insulin resistance induced by free fatty acids. Hepatobiliary Pancreat Dis Int 2010; 9:49-53.

25. Wu HT, Chen W, Cheng $\mathrm{KC}$, Yeh $\mathrm{CH}$, Shen KH, Cheng JT. Indomethacin activates peroxisome proliferator-activated receptor $\gamma$ to improve insulin resistance in cotton pellet granuloma model. Hormone and Metabolic Research 2010; 42(11):77580.

26. Strippoli GFM, Craig MC, Schena FP, Craig JC. Role of blood pressure targets and specific antihypertensive agents used to prevent diabetic nephropathy and delay its progression. J Am Soc Nephrol 2006;17(4 Suppl 2):S153-5.

27. Maione A, Navaneethan SD, Graziano G, Mitchell R, Johnson D, Mann JF, et al. Angiotensin-converting enzyme inhibitors, angiotensin receptor blockers and combined therapy in patients with microand macroalbuminuria and other cardiovascular risk factors: a systematic review of randomized controlled trials. Nephrol Dial Transplant. 2011; 26 (9):2827-47.
28. Barkin RL, Beckerman M, Blum SL, Clark FM, Koh EK, Wu DS. Should nonsteroidal anti-inflammatory drugs (NSAIDs) be prescribed to the older adult? Drugs Aging 2010;27(10):775-89.

29. Henz S, Maeder MT, Huber S, Schmid M, Loher M, Fehr T. Influence of drugs and comorbidity on serum potassium in 15000 consecutive hospital admissions. Nephrol Dial Transplant 2008;23(12):3939-45.

30. Guyton AC, Hall JE, editors. Renal regulation of potassium, calcium, phosphate, and magnesium; integration of renal mechanisms for control of blood volume an extracellular fluid volume. In: Textbook of medical physiology, $11^{\text {th }}$ ed., Elsevier Saunders, Elsevier Inc., Philadelphia, Pennsylvania 2006:365-382.

31. Bakris GL, Weir MR. Angiotensinconverting enzyme inhibitor-associated elevations in serum creatinine-is this a cause for concern? Arch Intern Med 2000;160(5):685-93.

32. Bouvy ML, Heerdink ER, Hoes AW, Leufkens HG. Effects of NSAIDs on the incidence of hospitalisations for renal dysfunction in users of ACE inhibitors. Drug Saf 2003;26(13):983-9.

33. Goswami SK, Jain S, Chudasama H, Santani D. Potential pharmacodynamic drug-drug interaction between concomitantly administered lisinopril and diclofenac sodium: a call for appropriate management in hypertensive osteoarthritic patients. Drug Metab Drug Interact 2011;26(3):127-37.

34. Barkin RL, Beckerman M, Blum SL, Clark FM, Koh EK, Wu DS. Should nonsteroidal anti-inflammatory drugs (NSAIDs) be prescribed to the older adult? Drugs Aging 2010;27(10):775-89. 